\title{
REMARKS ON THE EPISTEMIC INTERPRETATION of PARACONSISTENT LOgic
}

\author{
NiCOLÁS LO GUERCIO \\ IIF-SADAF, CONICET, 642 Bulnes, Buenos Aires 1176, ARgEnTINA \\ nicolasloguercio@gmail.com \\ DAMIAN SZMUC \\ IIF-SADAF, CONICET, 642 Bulnes, Buenos Aires 1176, ARGENTINA \\ szmucdamian@gmail.com
}

\begin{abstract}
In a recent work, Walter Carnielli and Abilio Rodrigues present an epistemically motivated interpretation of paraconsistent logic. In their view, when there is conflicting evidence with regard to a proposition $A$ (i.e. when there is both evidence in favor of $A$ and evidence in favor of $\neg A$ ) both $A$ and $\neg A$ should be accepted without thereby accepting any proposition $B$ whatsoever. Hence, reasoning within their system intends to mirror, and thus, should be constrained by, the way in which we reason about evidence. In this article we will thoroughly discuss their position and suggest some ways in which this project can be further developed. The aim of the paper is twofold. On the one hand, we will present some philosophical critiques to the specific epistemic interpretation of paraconsistent logic proposed by Carnielli \& Rodrigues. First, we will contend that Carnielli \& Rodrigues's interpretation implies a thesis about what evidence rationally justifies to accept or believe, called Extreme Permissivism, which is controversial among epistemologists. Second, we will argue that what agents should do, from an epistemic point of view, when faced with conflicting evidence, is to suspend judgment. On the other hand, despite these criticisms we do not believe that the epistemological motivation put forward by Carnielli \& Rodrigues is entirely wrong. In the last section, we offer an alternative way in which one might account for the epistemic rationality of accepting contradictions and, thus, for an epistemic understanding of paraconsistency, which leads us to discuss the notion of diachronic epistemic rationality.
\end{abstract}

Keywords: Logics of Formal Inconsistency • epistemic justification • evidence.

\section{Introduction}

One of the aims of this paper is to present some philosophical critiques to the epistemic interpretation of paraconsistent logic proposed by Walter Carnielli and Abilio Rodrigues in e.g. (2015). In their own words, their intention is to provide an epistemic interpretation of paraconsistent logics that is analogous to the Brouwer-Heyting-Kolmogorov interpretation of intuitionistic logic. The main claim of this pur- 
ported interpretation is that when there is conflicting evidence with regard to a proposition $A$, i.e. when there is both evidence in favor of $A$ and evidence in favor of $\neg A$, both $A$ and $\neg A$ should be accepted without thereby accepting any proposition $B$ whatsoever. Carnielli \& Rodrigues maintain that this is what actual scientists do, or at least what they have done in the past. ${ }^{1}$ More generally, in Carnielli \& Rodrigues's (2015, p.13) terms:

As we have seen, 'evidence that $A$ is true' is understood as 'reasons for believing that $A$ is true', while 'evidence that $A$ is false' means 'reasons for believing that $A$ is false'. We must distinguish the presence of evidence that $A$ is false from the absence of evidence that $A$ is true. Let ' $A$ is false' be represented by $\neg$ A. Accordingly:

'A holds' means 'there is evidence that $A$ is true'

'A does not hold' means 'there is no evidence that $A$ is true'

' $\neg A$ holds' means 'there is evidence that $A$ is false'

' $\neg A$ does not hold' means 'there is no evidence that $A$ is false'

The following four scenarios are possible:

1. No evidence at all: both $A$ and $\neg A$ do not hold

2. Only evidence that $A$ is true: $A$ holds and $\neg A$ does not hold

3. Only evidence that $A$ is false: $A$ does not hold and $\neg A$ holds

4. Conflicting evidence about $A$ : both $A$ and $\neg A$ hold

In order to give a formal shape to their claim, the authors design a logic called "Basic Logic of Evidence". Reasoning within this system is supposed to mirror and, thus, should be constrained by the way in which we reason about evidence. We will argue that their position faces some philosophical problems. However, since we do not believe that the epistemological motivation put forward by Carnielli \& Rodrigues is entirely wrong, we will suggest-following their approach-that there is an alternative way in which one might account for the epistemic rationality of accepting contradictions within scientific contexts and, thus, for an epistemic understanding of paraconsistency.

The paper is structured as follows. In Section 2 we make some preliminary remarks concerning the way in which we understand Carnielli \& Rodrigues position. In section 3, we argue that in its present form the epistemic interpretation of paraconsistency implies Extreme Permissivism, a thesis about what the evidence rationally justifies to accept or believe which is controversial among epistemologists. Later, in Section 4, we offer some reasons to argue that Extreme Permissivism is in fact problematic. Furthermore, we argue that what conflicting evidence actually supports is 
not accepting a pair of contradictory propositions but suspending judgment. In Section 5 we claim that despite these criticisms, the original epistemic motivation put forward by Carnielli \& Rodrigues might be on the right track. We offer an alternative way of accounting for the rationality of paraconsistent reasoning within scientific contexts, focusing on the distinction between synchronic and diachronic epistemic rationality. We end, in Section 6, with some concluding remarks.

\section{Preliminaries}

Before jumping into the main arguments, it will be worth making some clarifications concerning the way in which we understand Carnielli \& Rodrigues's view.

Carnielli \& Rodrigues avoid talking about belief of contradictions. Instead, they prefer to talk about acceptance, e.g. "a paraconsistent logic may be concerned with a notion weaker than truth, and it is precisely this weaker notion that allows an intuitive and plausible understanding of the acceptance of contradictions in some contexts of reasoning." (Carnielli \& Rodrigues 2015, p.2, our emphasis).

We take it to be fairly clear that they mean rational acceptance. But acceptance, even if rational, is a slippery business. Rational acceptance might be in principle constrained by more than just evidence considerations, or even entirely unconstrained by these. In Stalnaker's terms, for example, "to accept a proposition is to treat it as true for some reason" (Stalnaker 2002, p.716). Thus, one might accept something (treat it as true) because one believes it is true, because there is sufficiently strong evidence in support of that proposition, because one wants to see where the argument goes or just because one wants it to be true (as in cases of wishful thinking). All these might be cases of rational acceptance, although in a different sense of rationality. Not all of these senses are epistemic (some are pragmatic, prudential, etc.) and, therefore, not all of them give place to rational acceptance as an epistemic phenomenon.

Now, we take it that in maintaining that the acceptance of contradictions should be understood in terms of conflicting evidence, Carnielli \& Rodrigues are not thinking in a pragmatic or prudential notion of rational acceptance but in the notion of epistemically rational or epistemically justified acceptance. There are different views concerning what makes an attitude epistemically rational or justified. ${ }^{2}$ One might claim, for example, that an attitude is justified only if it is the output of a reliable belief-forming process. Now, in several places Carnielli \& Rodrigues emphasize the role of evidence in acceptance both of simple sentences and contradictions:

The basic idea is that the acceptance of a pair of contradictory propositions $A$ and $\neg A$ does not need to mean that both are true. Rather, we may understand it as some kind of 'conflicting information', namely, that there is conflicting evidence about $A$. Evidence for $A$ is understood in broad terms as reasons for 
believing that $A$ is true. Notice that evidence is a weaker notion than truth, in the sense that if one knows that a proposition $A$ is true, one has evidence that $A$ is true, but the converse obviously does not hold. (Carnielli \& Rodrigues 2015, p.2, our emphasis).

Since we take it that they are concerned with epistemically rational acceptance, and they cash out this notion in terms of evidence, we believe it is reasonable to think that they are assuming a form of evidentialism, viz. the view that evidence fully determines what is rational to accept (or believe, or what one is justified to believe) (See Conee \& Feldman 2004): ${ }^{3}$

Evidentialism Doxastic attitude $D$ toward proposition $p$ is epistemically justified for $S$ at $t$ if and only if having $D$ toward $p$ fits the evidence $S$ has at $t$. (Conee \& Feldman 2004, p.83)

Although Conee \& Feldman originally lay out the view in terms of belief, we think that Carnielli \& Rodrigues have something analogous in mind for the case of epistemically rational acceptance. Put differently, we will assume that whenever some body of evidence supports a proposition, it is rational for an agent in possession of that evidence to accept that proposition, and conversely, whenever it is epistemically rational for an agent to accept a proposition, it is because she is in possession of evidence that supports that proposition. Thus, we take Carnielli \& Rodrigues's point of view to be that there are cases in which it is epistemically rational to accept a pair of contradictory propositions, and this is solely in virtue of the possession of conflicting evidence regarding the matter. As a consequence, we assume that epistemically rational acceptance must respect constraints analogous to those of epistemically rational belief understood in evidentialists terms.

This is, we think, one of the most interesting ways to read Carnielli \& Rodrigues's proposal. If one allowed other non-evidential factors to play a role in determining what is rational to accept, their view that it is conflicting evidence what makes it sometimes rational to accept a contradiction would have to be revised.

\section{The road to Extreme Permissivism}

The "Basic Logic of Evidence" (BLE, for short) recently developed and defended by Carnielli \& Rodrigues aims to incarnate their epistemic understanding of the acceptance of contradictions in several contexts of reasoning, especially within scientific research. Since the main motivation is epistemological, it is worth examining how their system fares with respect to other plausible epistemological restrictions, in particular in relation with some theses concerning the correct reasoning about evidence. 
In this and the following section we will argue that the proposed epistemological understanding of contradictions faces some philosophical troubles. Accepting BLE's rules for conjunction, and some highly intuitive principles ${ }^{4}$ for reasoning with evidence, commits Carnielli \& Rodrigues with a position called Extreme Permissivism, a controversial view about evidence. Thus, anyone who embraces the logic of evidence BLE with this epistemic motivations could be targeted with these critiques against Extreme Permissivism.

In what follows we begin by presenting and distinguishing Extreme Permissivism both from moderate forms of permissivism and from Carnielli \& Rodrigues's view. We then go on showing how anyone who embraces BLE with these motivations is committed to Extreme Permissivism.

Let us start, then, by presenting Extreme Permissivism. In the last years, a significant amount of epistemological work has been devoted to the question of how wide the range of doxastic attitudes rationally permitted by the evidence can be (see Feldman 2007, White 2005, Douven 2009, Kelly 2013, Ballantyne et. al. 2012, Lee 2013, Schoenfield 2014). In this regard, some have favored the so-called Uniqueness thesis concerning evidence (White 2005, Feldman 2007, Christensen 2016, Matheson 2010). The Uniqueness thesis contends that given any body of evidence and any proposition, there can only be one doxastic attitude toward that proposition that is rationally permitted by the evidence:

Uniqueness Necessarily, for any proposition $p$ and body of evidence $e$, there is some doxastic attitude $D$ such that, for any subject $S$, if $e$ is $S$ 's total evidence, then it is rational for $S$ to have $D$ towards $p$ and irrational for $S$ to have any contrary doxastic attitude towards $p$. (Lee 2013, p.164)

On the other hand, there are those who favor a permissive view about evidence (Kelly 2013, Douven 2009, Schoenfield 2014). ${ }^{5}$ Permissivism is the view that results from rejecting Uniqueness. According to Permissivism, there are some cases in which the evidence rationally permits more than one doxastic attitude toward a proposition. ${ }^{6}$ We will now distinguish three forms of permissivism: Extreme Permissivism, moderate variants of permissivism and, finally, a stronger variant of Extreme Permissivism, embraced by Carnielli \& Rodrigues.

The view discussed by White (2005), that he dubbed Extreme Permissivism, maintains that there are possible cases in which not only the evidence permits different doxastic attitudes toward a proposition but it allows radically incompatible ones, i.e. there are cases in which a single body of evidence makes it rational either to accept (or believe, or disbelieve, and so on) $A$ or to accept $\neg A$, for some proposition $A$.

More moderate forms of permissivism typically assume a subjectivist approach to evidence support (see Douven 2009). By way of illustration, consider Subjective Bayesianism. According to Subjective Bayesianism, $k$ is evidence for $A$ if and only if 
the conditional probability of $A$ given $k$ is greater than the unconditional probability of $A$. Most importantly, according to Subjective Bayesianism there is no unique, objectively correct probability function that determines what the evidence supports (although that does not mean that all probability functions are acceptable). So according to Subjective Bayesianism the same evidence might support belief in $A$ (or some degree of confidence in $A$ that is sufficiently high as to license belief in $A$ ) relative to some subjective probability function $\operatorname{Pr}_{1}$, and belief in $\neg A$ (or some degree of confidence in $\neg A$ that is sufficiently high as to license belief in $\neg A$ ) relative to a different subjective probability function $P r_{2}$. In this sense, according to subjective bayesians the evidence might be permissive, for it might support $A$ relative to one probability function and $\neg A$ relative to a different one.

In the same line, Schoenfield 2014 defends permissivism by claiming that a body of evidence might make it rational to believe $A$ relative to some epistemic standard and $\neg A$ relative to a different epistemic standard, and Kelly 2013 argues that different doxastic attitudes might be rational for different subjects that give priority to different epistemic goals. ${ }^{7}$ It is important to note that moderate forms of permissivism deny that there are cases in which the evidence makes it rational to accept either $A$ or $\neg A$ relative to the same epistemic standard, let alone accept both of them. Moreover, Carnielli \& Rodrigues do not make any explicit mention of epistemic standards or the idea that evidential support is a three-place relation that involves a subjective component, so we take it that they do not endorse any of these forms of moderate permissivism.

Finally, there is a stronger form of Extreme Permissivism, implicitly embraced by Carnielli \& Rodrigues which entails that there are possible cases in which not only the evidence makes it rational either to accept $A$ or to accept $\neg A$, for some proposition $A$, but also to accept $A \& \neg A$, that is, to accept $A$ and to accept $\neg A$ at the same time. Let us notice that this stronger variant of permissivism entails Extreme Permissivism, but not the other way around. Extreme Permissivists may agree that some body of evidence makes it rational either to accept $A$ or to accept $\neg A$, but refrain from admitting that it is rational to accept $A$ and to accept $\neg A$, at the same time.

Having clarified this, in what follows we present our reasons to argue that Carnielli \& Rodrigues's understanding of BLE entails Extreme Permissivism. We begin by assuming with Carnielli \& Rodrigues that there is some proposition $A$ such that there is evidence $k$ supporting $A$ and there is evidence $k^{\prime}$ supporting $\neg A$. From this we will arrive at a position according to which some piece of evidence shall be seen as supporting (i.e. making it rational to accept) either a proposition or its negation, i.e. Extreme Permissivism. ${ }^{8}$

First, when treating the rule for the Introduction of Conjunction, Carnielli \& Rodrigues claim that "if $k$ and $k^{\prime}$ are evidence, respectively, for $A$ and $B, k$ and $k^{\prime}$ together 
constitute evidence for $A \& B$ " (Carnielli \& Rodrigues 2015, p.15, our emphasis). In these cases, for any $k$ and $k$ ', let the body of evidence constituted by $k$ and $k^{\prime}$ 'together" be denoted by $k+k^{\prime}$.

In case there is conflicting evidence, $k$ and $k^{\prime}$, with regard to $A$, the rule for the Introduction of Conjunction (Carnielli \& Rodrigues 2015, p.14) yields the result that $k$ and $k^{\prime}$ together support the conjunction $A \& \neg A$. Given our terminological remarks, we will refer to $k$ and $k^{\prime}$ together as $k+k^{\prime}$ and we might as well denote $k+k^{\prime}$ by $k^{*}$. Our first conclusion is, thus, that the evidence $k^{*}=k+k^{\prime}$ supports $A \& \neg A .{ }^{9}$

Secondly, we can infer that the evidence $k^{*}=k+k^{\prime}$ supports $\neg(A \& \neg A)$, as follows. On the one hand, from our supposition that evidence $k$ supports $A$, we infer by the rule for the Introduction of Double Negation (Carnielli \& Rodrigues 2015, p.18) that evidence $k$ supports $\neg \neg A .{ }^{10}$ From this, by the rule for the Introduction of Negated Conjunction ${ }^{11}$ (Carnielli \& Rodrigues 2015, p.17), we infer that evidence $k$ supports $\neg(A \& \neg A)$. On the other hand, from our supposition that evidence $k^{\prime}$ supports $\neg A$, we infer by the rule for the Introduction of Negated Conjunction that evidence $k^{\prime}$ supports $\neg(A \& \neg A)$.

We, thus, arrive at the interesting fact that both the evidences $k$ and $k^{\prime}$ support $\neg(A \& \neg A)$. Still, what we need to establish our purported conclusion is that $k$ and $k^{\prime}$ together support $\neg(A \& \neg A)$. We might arrive at this conclusion via two highly intuitive paths.

The first available path to conclude that $k^{*}$ supports $\neg(A \& \neg A)$ is via what we take to be a sound principle about evidence: if evidence $k_{1}$ supports $C$ and evidence $k_{2}$ supports $C$ too, then $k_{1}$ and $k_{2}$ together should support $C$. If one thinks that this is in fact true, then our desired conclusion is firmly established. ${ }^{12}$

To better see the point, imagine that this principle does not hold. Then it should be possible that after finding new positive evidence in favor of a proposition that I already accept I must lower my confidence in such proposition, in the limit case, even abandon it. This would be clearly irrational from an epistemic point of view. Moreover, if this principle were not valid it would be hard to explain why we tend to search for new evidence in favor of our theories: in fact, we do it because we think that new evidence will increase the rationality of holding those theories.

To consider another possible objection to this principle, assume one admits that evidence $k_{1}$ supports $C$, that evidence $k_{2}$ supports $C$ and that evidence $k_{2}$ supports $\neg C$ too. ${ }^{13}$ Then one might refrain from admitting that $k_{1}$ and $k_{2}$ together support $C$, by saying that the conflicting pieces of evidence $k_{1}$ and $k_{2}$ together actually support the suspension of judgment about $C$, not its acceptance (a view that, as we will discuss, most epistemologists share). But, then, this will speak against Carnielli \& Rodrigues motivation for embracing BLE: they argue that in the face of conflicting evidence about $C, C$ should still be accepted. Therefore, if this principle is denied on the foregoing explicit grounds, then the very motivation for BLE is undermined. 
Therefore, given that the previous considerations are not solid for us, we think it is more than philosophically justified to stick to this principle.

Thus, in our case, given that evidence $k$ supports $\neg(A \& \neg A)$ and evidence $k^{\prime}$ supports $\neg(A \& \neg A)$, we infer that evidence $k$ and $k^{\prime}$ together support $\neg(A \& \neg A)$. Via this path, our second conclusion is, therefore, established: $k^{*}=k+k^{\prime}$ supports $\neg(A \& \neg A)$.

The second available path to conclude that $k^{*}=k+k^{\prime}$ supports $\neg(A \& \neg A)$ involves a form of Contraction or Idempotence for Conjunctions (see e.g. Dunn and Restall 2002). We take it to be a sound principle about evidence that if evidence $k_{1}$ supports $C \& C$, then evidence $k_{1}$ supports $C . .^{14}$

One might, however, challenge the soundness of this principle about evidence, by arguing, for example, that $C \& C$ differs from $C$ in some relevant respect. Typically, logicians that reject the structural rule of Contraction back the claim that (for some conjunction "\&") the propositions $C \& C$ and $C$ are different, highlighting that the number of occurrences of a certain proposition e.g. $C$ matters. Thus, $C$ is not the same as $C \& C$ and neither is $C \& C$ equal to $(C \& C) \&(C \& C)$. However, this seems not to be the case when reasoning about evidence, for when we are in possession of some evidence, we can use it as many times as we want, i.e. it may occur as many times as we want in our reasoning. Therefore, given that the previous considerations against this principle are not solid for us, we think it is more than philosophically justified to stick to it.

Thus, in our case, the way to infer that $k^{*}=k+k^{\prime}$ supports $\neg(A \& \neg A)$ will be the following. By the previous reasoning, we have that evidence $k$ supports $\neg(A \& \neg A)$ and that evidence $k^{\prime}$ supports $\neg(A \& \neg A)$. Using the rule for the Introduction of Conjunction, we arrive at the fact that $k$ and $k^{\prime}$ together support $\neg(A \& \neg A) \& \neg(A \& \neg A)$. Now, by Contraction or Idempotence we arrive at the fact that evidence $k$ and $k^{\prime}$ together support $\neg(A \& \neg A)$. Again, via this alternative path, we establish our desired conclusion: $k^{*}=k+k^{\prime}$ supports $\neg(A \& \neg A)$.

One last clarification: according to Carnielli \& Rodrigues's view, if there is conflicting evidence $k$ and $k^{\prime}$ regarding $A$, this entails that there is also evidence (i.e. $k^{*}=k+k^{\prime}$ ) that supports some proposition $A \& \neg A$ and that also supports its negation $\neg(A \& \neg A)$. Under the evidentialist assumption above mentioned, this means that according to Carnielli \& Rodrigues sometimes it is rational to accept a pair of contradictory propositions. It should be noted that Extreme Permissivism does not contend that there are cases in which the evidence makes it rational to accept at the same time a pair of contradictory propositions. It only maintains that the evidence sometimes makes it rational either to accept one of them or the other. So Carnielli \& Rodrigues interpretation of BLE entails Extreme Permissivism, but not the other way around.

In any case, we take it to be firmly established that by following Carnielli \& Ro- 
drigues's understanding of BLE plus some plausible assumptions, we arrive at a position according to which some bodies of evidence support both a proposition and its negation, thus it makes it rational to accept either one or the other. This is in fact what Extreme Permissivism (see e.g. White 2005) maintains.

\section{Philosophical concerns about Extreme Permissivism}

In this section we will discuss Extreme Permissivism. First, we will present an argument against it and, a fortiori, against the previously discussed epistemic motivation of paraconsistent logic. In addition, we will present a direct argument against Carnielli \& Rodrigues particular epistemic interpretation of paraconsistency, specifically against the idea that conflicting evidence can make it epistemically rational to accept a contradiction.

So far we have argued that Carnielli \& Rodrigues reasons to adopt BLE commits their view with Extreme Permissivism. We believe this is bad news for the present form of their account, since Extreme Permissivism is controversial, even among those who favor a permissive view about evidence. However, we do not claim that it affects all possible variants of the epistemic understanding of paraconsistency, as we will show in Section 5.

To see the point, we will present what we think is the most pressing argument for rejecting Extreme Permissivism. The argument maintains that acceptance in the face of extremely permissive evidence is arbitrary, hence irrational. ${ }^{15}$ Suppose that the total evidence is extremely permissive. That means that it supports either accepting $A$ or accepting $\neg A$, to the point that holding either would be equally rational. Additionally, imagine that you are aware of the fact that the evidence is extremely permissive. Then, how do you 'decide' what to accept? If you accept $A$, you will be aware that there is an equally rational path that would have led you to accept $\neg A$, and viceversa. In other words, in accepting $A$ you are aware that you could just change your decision to $\neg A$ and still be rational. Now, assuming evidentialism (as Carnielli \& Rodrigues do), from the point of view of epistemic rationality, you should accept only what is supported by the evidence. This is because we believe that following the evidence is your best option in pursuing the truth. But, from the point of view of pursuing the truth, what is the difference, in the case at hand, between basing your acceptance on your examination of the evidence and deciding by flipping a fair coin? If both $A$ and $\neg A$ are rationally permitted by the total evidence then it seems that whatever you end up accepting will be okay. Moreover, you are aware of this fact. So the decision will be inevitably based either on non-evidential factors (thus, becoming epistemically irrational) or it will be plainly arbitrary (as we said, you could just flip a coin in order to choose what to accept).

Put differently, as epistemic agents, we seek to be epistemically rational in ac- 
cepting propositions, i.e. we seek to accept propositions that are supported by the available evidence. The reason is that we believe that doing that will lead us more often than not to accept true propositions. But in a case in which the total evidence makes it equally rational both to accept $A$ and to accept $\neg A$, our chances of accepting a true proposition by following the evidence are just the same as those of accepting a true proposition by following non-evidential factors or just by flipping a fair coin. In light if this arbitrariness, we conclude that accepting either $A$ or $\neg A$ in the face of extremely permissive evidence would be epistemically irrational. In consequence, we must reject Extreme Permissivism. Since, as we showed, Carnielli \& Rodrigues's position plus some plausible assumptions entails Extreme Permissivism, this is an argument, a fortiori, against their view.

It might be objected that defenders of the epistemic interpretation might use our point about arbitrariness on their behalf: it is precisely because choosing $A$ rather than $\neg A$ (or $\neg A$ rather than $A$ ) would be arbitrary, that accepting both $A$ and $\neg A$ is the only rational attitude when one is faced with conflicting evidence about $A$. Since it is equally rational both to accept $A$ and to accept $\neg A$, to end up accepting only one of them would be arbitrary: there would be a proposition that one does not accept (even if one does not explicitly reject it) that enjoys equal evidential support than another proposition one accepts. However, one might argue, accepting both of them would not be arbitrary in this sense. After all, on the one hand, in the imagined case both $A$ and $\neg A$ are to some extent supported by the evidence, and on the other hand, by accepting $A$ and accepting $\neg A$ one avoids the problem of not accepting a proposition that enjoys equal evidential support than other proposition one accepts. ${ }^{16}$

There are two problems with this answer. First, as we showed in the previous section, Carnielli \& Rodrigues' view plus some plausible assumptions entails Extreme Permissivism, i.e. they cannot escape the arbitrariness worry without either modifying their position in a way that does not entail Extreme Permissivism, or rejecting the plausible assumptions. In other words: their view entails that at least for some $A$ one can rationally accept only $A$ (or only $\neg A$ ), in the face of conflicting evidence. Even if they believe that accepting both (A and $\neg A$ ) would be better, what we showed in Section 3 is that they are committed to the view that accepting only one of them (either one of them, that is) is rational. Since they are committed to this form of Extreme Permissivism, their view is vulnerable to the arbitrariness objection. So the previous argument stands. The second problem is that, as we will argue next, there are reasons to maintain that in the face of conflicting evidence, in which both $A$ and $\neg A$ enjoy equal evidential support, epistemic rationality mandates suspension of judgment. ${ }^{17}$

Although it is natural to assume that some parts of a total body of evidence might support $A$ while other parts of it might support $\neg A$ (what Carnielli \& Rodrigues call conflicting evidence), it is incoherent to claim that the whole evidence makes it rational to accept both $A$ and $\neg A .{ }^{18}$ To see the point, it is important to note that any 
piece of evidence in support of $A$ is also evidence against $\neg A$, and viceversa. Carnielli \& Rodrigues' agreement with this point is made explicit, when they spell out the motivations for the rule of Introduction for Double Negation (2015, p.18). To put it differently, evidence in support of $A$ constitutes a rebutting defeater for $\neg A$, and viceversa (see Pollock 1987; 1999). ${ }^{19}$

With this in mind, one can construct an argument against this version of the epistemic interpretation of paraconsistency. Imagine that you possess evidence $k$ in favor of $A$ and evidence $k^{\prime}$ in favor of $\neg A$. There are three possible scenarios. First scenario: $k$ is stronger than $k^{\prime}$, so the total evidence, $k+k^{\prime}$, supports $A$. Second scenario: $k^{\prime}$ is stronger than $k$, so the total evidence, $k+k^{\prime}$, supports $\neg A$. Third scenario: $k$ and $k^{\prime}$ provide equally strong support, respectively, to $A$ and $\neg A$. Carnielli \& Rodrigues contend that in the latter case accepting either $A$ or $\neg A$ would be rational. Moreover, they maintain that accepting $A \& \neg A$ would also be rational. In contrast, we will argue below that in these cases the total evidence supports suspending judgment about whether $A$ is the case. If this is on the right track, either the total evidence supports $A$, or $\neg A$, or it supports suspending judgment regarding the matter. In any case, it cannot be that the total evidence supports both $A$ and $\neg A$, let alone $A \& \neg A$, even if the total body of evidence encompasses conflicting pieces of evidence. ${ }^{20}$

As we said earlier, evidence in support of $A$ defeats evidence for $\neg A$ and viceversa. In other words, evidence for $A$ is a reason for accepting $A$ but also a reason for not accepting $\neg A$, and the other way around. If $k$ and $k^{\prime}$ are equally good then $k$ defeats evidence $k^{\prime}$, while $k^{\prime}$ defeats evidence $k$. That means that $k$ and $k^{\prime}$ "neutralize" themselves: the support $k$ provides to $A$ is defeated by $k^{\prime}$, and the support that $k^{\prime}$ provides to $\neg A$ is defeated by $k$. So the reasons for and against accepting $A$ are balanced, and the same goes for $\neg A$. Thus, intuitively one should not accept either.

Not only this conclusion is intuitive: many epistemologists, both on the internalist and the externalist side, believe that there is a no-defeater condition on rationality or justification..$^{21}$ If this is on the right track, accepting $A$ is not epistemically rational due to the existence of defeating evidence, and accepting $\neg A$ is not epistemically rational for the same reason, thus it is not rational to accept neither. Therefore, it is not possible that a body of evidence, even if it encompasses equally strong evidence for $A$ and $\neg A$, makes it epistemically rational both to accept $A$ and to accept $\neg A$. If this is on the right track, Carnielli \& Rodrigues's specific epistemic interpretation of paraconsistency (which maintains that the rational thing to do when faced with conflicting evidence is to accept both $A$ and $\neg A$ ) is problematic.

We now consider a few possible objections to this first argument against Carnielli \& Rodrigues view. One might insist that in the face of equally strong conflicting evidence regarding $A$ and $\neg A$ one should not suspend judgment regarding $A$, but accept both. Carnielli \& Rodrigues remind us that scientists actually accept contradictions sometimes, so unless we are willing to maintain that they are being irrational, we 
must accept that in those cases accepting a contradiction is what the evidence supports. We will tackle this worry with more detail in the following section. We believe there is an alternative way of accounting for the rationality of scientists that accept contradictions. Here, we provide instead some additional considerations to support the point that equally strong conflicting evidence supports the suspension of judgment.

It is typically maintained that reaching the truth and avoiding the falsity are the two primary epistemic goals. ${ }^{22}$ Following the evidence is supposed to be a good way of achieving these goals: we typically assume that by following the evidence (instead of e.g. guessing, or believing what makes us happy) we will accept true propositions more often than not, as well as avoid accepting falsities more often than not. ${ }^{23}$ Now, in cases in which there is equally strong conflicting evidence, we claim, one must suspend judgment. What is the epistemic benefit of that? Of course, suspending judgment is not something that will contribute to the goal of accepting what is true. However, by suspending judgment one avoids accepting what is false. In the cases at hand, in which the evidence makes $A$ and $\neg A$ equally likely, following the evidence is not helpful in order to achieve the goal of accepting true propositions, so the best we can do is to avoid accepting false ones. By suspending judgment we do precisely that.

In contrast, it is not clear what would be the epistemic gain of accepting a contradiction. Accepting a contradiction not only does not contribute to the goal of accepting true things but it conspires against the goal of not accepting false ones, since by accepting a pair of contradictory propositions one is guaranteed to accept something false. ${ }^{24}$ If accepting contradictions does not contribute to achieving any of the primary epistemic goals, then it cannot be what epistemic rationality mandates.

Having presented some negative arguments against Carnielli \& Rodrigues, we will offer, in the following section, an alternative proposal for understanding the acceptance of contradictions in epistemic terms.

\section{Suggestions to develop an epistemic interpretation of paraconsistency}

In this section we elaborate a general worry about the desired epistemic interpretation of paraconsistent systems defended by Carnielli \& Rodrigues, and suggest some ways in which it might be further developed and strengthened. We contend that the scientific examples provided by Carnielli \& Rodrigues could be explained away without maintaining that conflicting evidence makes it epistemically rational to accept contradictions. In a nutshell, we argue that Carnielli \& Rodrigues overlook an important distinction between synchronic and diachronic rationality: once this distinction is taken into account, an alternative way of accounting for the rationality accepting 
a pair of contradictory propositions—and, therefore, for paraconsistency—becomes available.

Carnielli \& Rodrigues contend that empirical science, as a non-monotonic, selfcorrective epistemic endeavor, encompasses a lot of (provisional) internal inconsistencies, as well as inconsistencies between findings within different areas of research. In light of this, they argue that "In fact, in these cases the general argumentative framework is already paraconsistent because, obviously, in order to avoid a disaster, the principle of explosion cannot be valid." (Carnielli \& Rodrigues 2015, p.10). In order to make sense of this fact about reasoning in scientific contexts, they offer an epistemological interpretation of paraconsistent logic. Such interpretation consists in understanding the acceptance of a pair of contradictory propositions as supported by conflicting evidence. If this is on the right track, they have an explanation of why scientists sometimes rationally accept $A \& \neg A$, without the whole system becoming trivial.

In the previous section we argued that this position entails a view about evidence that is contentious. In this section, we will address an alternative way of explaining the rationality of the acceptance of contradictions within scientific inquiry, one that is not committed to the idea that sometimes conflicting evidence makes it rational to accept a contradiction.

Carnielli \& Rodrigues account for the fact that scientists sometimes accept contradictions by claiming that occasionally it is epistemically rational to do it, that is, by claiming that sometimes the evidence actually supports accepting contradictions. In the previous section we argued against this view. We defended that in cases of conflicting evidence epistemic rationality requires suspending judgment. However, if accepting contradictory propositions is not epistemically rational, the question arises: should we deem scientists that provisionally accept contradictory propositions as flatout irrational? Our answer is no. One might account for the rationality of the scientists' acceptance of contradictions in a different way.

An important point that we think has been slightly overlooked in Carnielli \& Rodrigues discussion is the distinction between synchronic and diachronic rationality (Conee \& Feldman 2004, p.189). Synchronic rationality concerns the propositions that the evidence makes rational to accept at a given moment. An agent that is rational in this sense must accept at a given time $t$ the propositions that are supported by the evidence she possesses at $t$, and nothing else. In the synchronic sense it is never epistemically rational to accept a contradiction. This is what we argued for in the previous section. In cases of conflicting evidence where the total evidence supports both propositions equally well, the rational thing to do in synchronic terms is to suspend judgment about the matter.

But occasionally scientists do not do that. Instead, they accept both propositions. Here is where diachronic rationality kicks in. Diachronic rationality concerns the goal 
of forming rational beliefs in the long term. In this sense, one might rationally accept now a statement that is not sufficiently supported by the current evidence, when this contributes to achieving one's epistemic goals in the future. This kind of acceptance might be rational on several grounds: a scientist might provisionally accept a statement just because there is no better explanation available, or because she has good reasons to believe that she will be able to collect further evidence that will provide the necessary support, or because it is easy to design experiments to test whether it is true or not, among many other reasons. So in this sense, it might be diachronically rational to accept a pair of contradictory propositions, although not synchronically rational-that is, even if accepting the contradiction is not supported by the currently available evidence.

Let us go back to the cases of conflicting evidence. Notwithstanding the fact that suspending judgment in those cases (in the sense of not accepting neither $A$ nor $\neg A$ ) is the right thing to do in synchronic terms, it is clear that it is not a good option in diachronic terms, for it would mean suspending the investigation. Abandoning acceptance of both conflicting statements means closing down the possibility of continuing the inquiry in order to find out which one is correct, that is, it blocks the possibility of finding new evidence, designing crucial experiments in order to decide between them, and so on. So, in order to continue the research, scientists might provisionally accept both propositions and see where each of them leads, with the hope of eventually being able to decide, in virtue of new evidence, novel experiments or fresh theoretical arguments which proposition must go and which must stay.

In fact, if scientists accepted contradictions in the synchronic sense, that is, if they accepted that the evidence supports $A \& \neg A$, it would be hard to make sense of the fact that they try by every mean to eliminate the contradiction: they search for further evidence that will be able to settle the matter either for $A$ or $\neg A$, or they try to formulate crucial experiments to decide.

So acceptance of a contradiction is epistemically irrational in the synchronic sense, but that does not mean that scientists are irrational, for accepting a contradiction might be rational in the diachronic sense. When scientists accept a contradiction, all that is needed in order to avoid disaster is to note that the criterion of rationality in play in those occasions is diachronic, not synchronic. So, there might be reasons for provisionally accepting such pair of contradictory propositions, for this allows scientists to continue the investigation with the idea that it might produce an epistemic benefit in the long term. However, this proposal would imply a slight modification of Carnielli \& Rodrigues's epistemic motivation of paraconsistency. On this view although it is epistemically rational to accept a contradiction, this is not because the currently conflicting evidence actually supports this acceptance, but because it will be epistemically beneficial in the long run. 


\section{Conclusion}

In this article we discussed Carnielli \& Rodrigues's epistemological interpretation of paraconsistency. We proceeded first by presenting their view. As we understand it, Carnielli \& Rodrigues claim that in some contexts of reasoning, e.g. scientific contexts, the acceptance of a pair of contradictory propositions does not imply the acceptance of any proposition $B$ whatsoever. In turn, they claim that the rationality underlying the acceptance of contradictions is epistemic. In other words, they maintain that sometimes the available evidence makes it rational to accept both $A$ and to accept $\neg A$.

We argued against this position in two steps. First, we showed that Carnielli \& Rodrigues position entails Extreme Permissivism, the view that some bodies of evidence support accepting either $A$ or $\neg A$, for some $A$. Then, we showed that Extreme Permissivism has contentious consequences, viz. either accepting $A$ or $\neg A$ in the face of Extremely Permissive evidence is arbitrary, since the decision must be based either on non-evidential factors or on mere chance. Thus, either believing $A$ or $\neg A$ in the face of extremely permissive evidence is epistemically irrational.

Second, we contended that the rational attitude, when confronted with conflicting evidence regarding $A$ and $\neg A$ is the suspension of judgment. In support of this position we claimed that suspending judgment contributes to one of the two primary epistemic goals, namely, avoid accepting false propositions. In contrast, believing a contradiction has no apparent epistemic gain.

Finally, we showed that it is possible to provide an alternative account of the rationality underlying the acceptance of contradictions within scientific contexts. We believe that Carnielli \& Rodrigues would benefit from adopting the distinction between synchronic and diachronic rationality. While the former concerns the attitudes that best fit the currently available evidence, the latter has to do with being rational in the long term. If this is on the right track, scientists sometimes provisionally accept contradictions because e.g. it allows them to continue investigation in order to gather more evidence, design experiments that might be able to decide the matter, or come up with new theoretical arguments that might tip the scales in one or other direction.

\section{References}

Ballantyne, N.; Coffman, E. J. 2012. Conciliationism and uniqueness. Australasian Journal of Philosophy 90(4): 657-670.

Bergmann, M. 1997. Internalism, externalism and the no-defeater condition. Synthese 110(3): 399-417.

Burge, T. 1993. Content preservation. The Philosophical Review 102(4): 457-488.

Priest, G.; Berto, F. 2017. Dialetheism. In: E. N. Zalta (ed.) The Stanford Encyclopedia of Philosophy. URL: https://plato.stanford . edu/archives/spr2017/entries/ dialetheism/ 
Carnielli, W.; Rodrigues, A. 2015. A logic for evidence and truth. CLE e-Prints 15(5): 1-13. Christensen, D. 2016. Conciliation, Uniqueness and Rational Toxicity. Noûs 50(3): 584-603. Conee, E.; Feldman, R. 2004. Evidentialism: Essays in epistemology. Clarendon Press.

Dougherty, T. 2011. Evidentialism and its Discontents. Oxford Un. Press.

Douven, I. 2009. Uniqueness revisited. American Philosophical Quarterly 46(4): 347-361.

Dunn, J. M.; Restall, G. 2002. Relevance logic. In: D. Gabbay; F. Guenther (eds.) Handbook of Philosophical Logic Volume 6, pp.1-128. Dordrecht: Kluwer.

Feldman, R. 2007. Reasonable Religious Disagreements. In: L. Antony (ed.) Philosophers Without Gods: Meditations on Atheism and the Secular, pp.194-214. Oxford University Press.

Humberstone, L. 2011. The Connectives. MIT Press.

Kelly, T. 2013. Evidence can be permissive. In: M. Steup; J. Turri; E. Sosa (eds.) Evidence can be permissive, pp.298-312. Wiley-Blackwell.

Kvanvig, J. 2005. Truth is not the Primary Epistemic Goal. In: M. Steup; J. Turri; E. Sosa (eds.) Contemporary Debates in Epistemology, pp.285-296. Wiley-Blackwell.

Lee, M. 2013. Conciliationism without Uniqueness. Grazer Philosophische Studien 88(1): 161188.

Matheson, J. D. 2010. Dealing with disagreement: uniqueness and conciliation. $\mathrm{PhD}$ thesis, University of Rochester.

Pollock, J. L. 1987. Defeasible reasoning. Cognitive Science 11(4): 481-518.

- 1999. Contemporary theories of knowledge. Rowman \& Littlefield

Rorty, R. 1995. Is truth a goal of enquiry? Davidson vs. Wright. The Philosophical Quarterly 45(180): 281-300.

Schoenfield, M. 2014. Permission to believe: Why permissivism is true and what it tells us about irrelevant influences on belief. Noûs 48(2): 193-218.

Stalnaker, R. 2002. Common ground. Linguistics and Philosophy 25(5): 701-721.

White, R. 2005. Epistemic permissiveness. Philosophical Perspectives 19(1):445-459.

Wright, C. 2004. Warrant for nothing (and foundations for free)? Aristotelian Society Supplementary Volume 78(1): 167-212.

\section{Notes}

${ }^{1}$ They quote the particular cases of the well known incompatibility between the classical, Newtonian mechanics and Maxwell's theory of electromagnetic fields, later solved by Einstein's work in his special theory of relativity. They say, with regard to this: "We want to call attention to the fact that the general logical framework Einstein was working in was not classical. He had two different theories at hand, classical mechanics and the theory of the electromagnetic field that, when put together, yielded a non-explosive contradiction. Later, according to the special theory of relativity, the 'contradiction' disappeared." (Carnielli \& Rodrigues 2015, p.13).

${ }^{2}$ We will use 'rational' and 'justified' (in the epistemic sense) interchangeably in this context. ${ }^{3}$ To be sure, not everybody in the literature agrees on this (see e.g. Dougherty 2011 for discussion).

${ }^{4}$ Let us highlight that the assumptions we refer to are not only highly plausible (and indeed, might have been addressed implicitly by Carnielli \& Rodrigues), but also are such that, if 
denied, would leave Carnielli \& Rodrigues's argumentation in very bad shape. When explicitly using these assumptions, we will argue in favor of their plausibility and discuss the critical results of denying them.

5 Uniqueness and Permissivism can be formulated either within an all-or-nothing view of belief, where the only doxastic attitudes allowed are belief, disbelief and suspension of judgment, or within a graded framework. For simplicity's sake, we will proceed by assuming an all-or-nothing view and making the necessary clarifications when needed.

${ }^{6}$ There is another way of denying Uniqueness, viz. one might contend that some bodies of evidence do not support any doxastic attitude toward some propositions. We do not know of any defense of this position, so hereinafter we will focus on the aforementioned view.

7 The same could be said for the case of a single individual who ascribes to different epistemic standards at different times.

8 Notice that up to this point, Carnielli \& Rodrigues account is not identifiable with Extreme Permissivism. That is to say, accepting that there is conflicting evidence with regard to a proposition $A$ is not the same as saying that the same piece of evidence supports both accepting $A$ and $\neg A$, for the acceptance of conflicting evidence with regard to $A$ is assumed to imply that evidence $k$ in favor of $A$ and evidence $k^{\prime}$ in favor of $\neg A$ are such that $k$ and $k^{\prime}$ are allowed to be different. Extreme Permissivism, on the other hand, requires the evidence in favor of $A$ and the evidence in favor of $\neg A$ to be the same piece of evidence.

${ }^{9}$ It might be thought that from the mere fact that there is evidence $k^{*}$ for $A \& \neg A$ one could conclude, by the rule of Conjunction Elimination, that $k^{*}$ is evidence for $A$, and also that $k^{*}$ is evidence for $\neg A$. Were this the case, just by having conflicting evidence for $A$, we would arrive at Extreme Permissivism. Unfortunately, this move is not available. To see this, notice that in the epistemic understanding of the logic BLE, the rule of Conjunction Elimination ensures that if evidence $k_{1}$ supports $A \& B$, then there is some evidence $k_{2}$ contained in $k_{1}$ that supports $A$, and there is some evidence $k_{3}$ contained in $k_{1}$ that supports $B$ (which is, in fact, analogous to the case of intuitionistic logic and the BHK interpretation, replacing evidence for proof), but nothing else is assumed with regard to $k_{2}$ and $k_{3}$. Especially, it is not assumed that they are identical. In the case in question, from the fact that $k^{*}$ is evidence for $A \& \neg A$, by the rule of Conjunction Elimination, it only follows that some evidence $k^{\prime \prime}$ contained in $k^{*}$ is evidence for $A$, and that, respectively, some evidence $k^{\prime \prime \prime}$ contained in $k^{*}$ is evidence for $\neg A$. However, from these two existential claims about the evidence for $A$ and the evidence for $\neg A$, it does not follow that $k^{\prime \prime}$ and $k^{\prime \prime \prime}$ are identical, i.e. we have not arrived at Extreme Permissivism. (We would like to thank an anonymous referee for Principia for discussion on this matter).

10 The rule for the Introduction of Double Negation allows to infer $\neg \neg A$ from $A$, for every formula $A$, see Carnielli \& Rodrigues 2015, p.18.

11 The rule for the Introduction of Negated Conjunction allows to infer $\neg(A \& B)$ from $\neg A$, for every formula $A, B$. See Carnielli \& Rodrigues 2015, p.17 (Additionally, recall that Conjunctions are commutative, i.e. $A \& B$ is identical to $B \& A$.)

12 If $k_{1}$ and $k_{2}$ are independent evidence in favor of $C, k_{1}$ together with $k_{2}$ (i.e. $k_{1}+k_{2}$ ) should add support to $C$ with respect to $k_{1}$ or $k_{2}$ alone. If $k_{1}$ and $k_{2}$ are not independent, still $k_{1}+k_{2}$ should support $C$ to some extent.

13 Notice that this possible objection already assumes Extreme Permissivism as a premise, as it is required that $k_{2}$ supports $C$ and that evidence $k_{2}$ supports $\neg C$. 
${ }^{14}$ We might call this principle Contraction because inferring that $k_{1}$ supports $C$ from the fact that $k_{1}$ supports $C \& C$ resembles (i.e. it is not identical, but similar to) the behavior of the structural rule of Contraction, which allows to infer $C$ from the set $\{C, C\}$. Alternatively, it can be seen as a form of Idempotence for Conjunctions, since inferring that if $k_{1}$ supports $C$ $\& C$ it equally supports $C$ (and viceversa), resembles (again it is not identical, but similar to) the property of Idempotence for Conjunctions, which establishes that $C \& C$ and $C$ are equal. See e.g. Humberstone 2011.

15 The argument is adapted from White 2005.

${ }^{16}$ We thank an anonymous referee for Principia for drawing our attention to this point.

17 An anonymous referee for Principia has suggested a possible line of defense. Whenever one has evidence for a proposition $A$, the suggestion goes, one is not only justified in accepting $A$ but also entitled to accept $A$. Thus, whenever there is conflicting evidence for $A$, one is rationally entitled to accept both $A$ and $\neg A$ and cannot rationally accept only one of them. We do not think that this alternative, at least as it stands, has good prospects. Typically, epistemic entitlement is understood as an epistemic right, the right of a subject to believe a proposition in cases in which such proposition is not supported by the available evidence (Wright 2004, Burge 1993). Hence, accounting for the acceptance of contradictory propositions $A$ and $\neg A$ in terms of epistemic entitlement seems to be at odds with Carnielli \& Rodrigues project of motivating such acceptance on the basis of, precisely, the availability of supporting (conflicting) evidence about both $A$ and $\neg A$. To be sure, we are not claiming that the suggested defense is hopeless, but the idea of entitlement (epistemic or otherwise) should be further developed and the way in which it would help Carnielli \& Rodrigues project should be made more clear. 18 Here we are ignoring moderate forms of permissivism, which we discarded.

19 "If $M$ is a defeasible reason for $S$ to believe $Q, M^{*}$ is a rebutting defeater for this reason if and only if $M^{*}$ is a defeater (for $M$ as a reason for $S$ to believe $Q$ ) and $M^{*}$ is a reason for $S$ to believe $\neg Q$." (Pollock 1999, p.196).

20 One might ask: why then scientists sometimes do not suspend judgment but continue accepting incompatible hypothesis? Are they being irrational? We will tackle this worry in the following section.

${ }^{21}$ Since we are interpreting Carnielli \& Rodrigues's position in evidentialist terms, we would probably want to lay out the the no-defeater condition (NDC, for short) in internalist terms, along the following lines: (NDC) is satisfied by $S$ 's belief that $p$ if and only if $S$ does not believe (and would not upon reflection) that her belief that $p$ is defeated (Bergmann 1997, p.407). In a more externalist spirit, one might formulate the condition in a way that requires there being no true proposition such that if $S$ believed it she would (or should) also believe that her belief that $p$ is defeated. Notice that both would serve our purposes here, since the imagined scenarios are such that both antecedents are satisfied.

${ }^{22}$ As is always the case in philosophy, not everybody agrees on this. Some pragmatists like Rorty deny that truth is the primary goal of investigation (see Rorty 1995 for discussion). See also Kvanvig 2005.

${ }^{23}$ This is usually presented in terms of belief, not of acceptance. But since we are dealing with epistemically rational acceptance, we assume that it is guided by the same general epistemic goals.

${ }^{24}$ One might claim that there are true contradictions (that is the position embraced by dialetheists, e.g. Priest and Berto 2017), but Carnielli \& Rodrigues explicitly reject this view (2015, p.26, p.29). 